\title{
The Management of Ocular Allergy in Community Pharmacies in the United Kingdom
}

Mr Paramdeep Bilkhu*, Prof James S. Wolffsohn*, Ms Deanna Taylor, Ms Emma Gibson, Mr Bhavik Hirani, Dr Shehzad A. Naroo*

*Ophthalmic Research Group, School of Life and Health Sciences, Aston University, Birmingham, B4 7ET

Email: bilkhups@aston.ac.uk

\section{Abstract}

Background: Ocular allergies frequently present in pharmacy practices. However, research into the actual management of ocular allergy in pharmacies is lacking.

Objective: To determine and quantify history and symptom questioning of a patient with presumed allergic conjunctivitis and management strategies employed by pharmacy staff in the UK

Method: A mystery shopper technique was used to simulate an episode of allergic conjunctivitis in 100 community pharmacies across the UK.

Results: The mean number of questions asked by pharmacy staff to the patient was $3.5 \pm 2.6$, with a range of $0-10$. The most common question was whether the patient had a history of allergies (45\%).Ninety-one percent advised on treatment, with the remaining $9 \%$ directly referring to the patient's general practitioner $(n=4)$ or pharmacist $(n=4)$, but only two to their optometrist. The most common treatment suggested was sodium cromoglycate $2 \%$ (50\%). However, many pharmacies advising treatment did not ask the patient's age (37\%), if they wore contact lenses (43\%), or gave dosage advice (43\%). Only $5 \%$ of pharmacies advised follow up and 14\% suggested visiting a general practitioner and $1 \%$ an optometrist if symptoms did not resolve with treatment. 
Conclusion: There is a need for improved ophthalmological training for pharmacy staff with respect to the management of allergic conjunctivitis

Abstract Length: 199 words

\section{Keywords}

Ocular allergy, allergic conjunctivitis, management, community pharmacy, United Kingdom (UK).

\section{Impact of Findings on Practice}

- Pharmacy staff should not attempt to differentially diagnose or manage allergic conjunctivitis without formal training or knowledge of the condition and eye disease.

- If the diagnosis of allergic conjunctivitis is in doubt, the patient must be referred to a member of staff with formal training or knowledge of the condition and eye disease or to the patient's optometrist.

- Careful consideration of the contraindications of ophthalmic preparations for allergic conjunctivitis is required if they are advised. 


\section{Introduction}

Ocular allergy affects approximately $20 \%$ of the allergy suffering population, representing a group of disorders that primarily affects the conjunctiva ${ }^{1}$.Ocular allergies are often underdiagnosed and under-treated, with ocular signs and symptoms considered part of other allergies rather than a distinct clinical entity ${ }^{2}$. Ocular allergies adversely impact on quality of life, school performance and work productivity and therefore pose a significant problem that needs to be managed appropriately ${ }^{2}$.

It has been estimated that $3 \%$ of patients consulting a pharmacist suffer from ocular allergy ${ }^{3}$. Combined with the availability of many anti-allergic preparations over the counter, pharmacists and pharmacy staff (under supervision of pharmacist) are required to differentially diagnose and manage ocular allergies based upon history and symptoms alone as they do not have access or training in using ophthalmic examination instruments. Furthermore, the signs and symptoms of ocular allergic disease may not be present at the time of a consultation ${ }^{1}$. However, research into the actual diagnosis and management of ocular allergy by pharmacists and pharmacy staff, has not been widely studied ${ }^{3}$.

\section{Objective}

This study aimed to determine and quantify the history and symptom questioning of a patient with presumed allergic conjunctivitis and management strategies employed by pharmacists and pharmacy staff in the UK. 


\section{Method}

A mystery shopper technique was used in 100 community pharmacies, selected using a randomised sampling technique, across the UK by 4 trained investigators, each visiting different practices alone. Pharmacies were a mixture of independent $(n=38)$ and chain $(n=62)$ practices. At each pharmacy the investigator approached the counter and when acknowledged by a staff member made the following opening statement:

"My brother's eyes are red and itchy. What would you recommend?"

The investigators answered the above questions only when asked but did not volunteer any information other than the opening statement. Immediately after leaving each pharmacy practice the consultation details were recorded in a table. It was expected that the mystery shopper should have been asked about their brother's age, current medications, whether they were a contact lens wearer, ocular history, history of allergies, past treatments I examinations and symptoms in more detail such as severity, duration, unilateral or bilateral, seasonality, pain and any discharge. ${ }^{4}$ Data was analysed using Microsoft Excel (Microsoft, USA). The study received Aston University ethics committee approval and the research conformed to the tenets of the Declaration of Helsinki. 


\section{$\underline{\text { Results }}$}

Out of a total of 100 pharmacy practices, $11 \%$ of consultations took place with a pharmacist, $21 \%$ with a pharmacy assistant or trainee dispenser, $8 \%$ with staff with ambiguous job titles, but in $60 \%$ of consultations it was not possible to determine the professional status as identification badges or labels were not visible or not worn.

The mean number of history and symptom questions asked by pharmacy staff was $3.5 \pm 2.6$, ranging from 0-10. Eleven percent of pharmacy staff asked additional questions - if there was blood in or on the eye; the patient had a cold; used cosmetics; the eyes felt gritty; any eyelid swelling; whether a personal computer was used frequently; and if a new laundry powder had been used. The questions asked by the pharmacy staff is presented in figure 1 . 


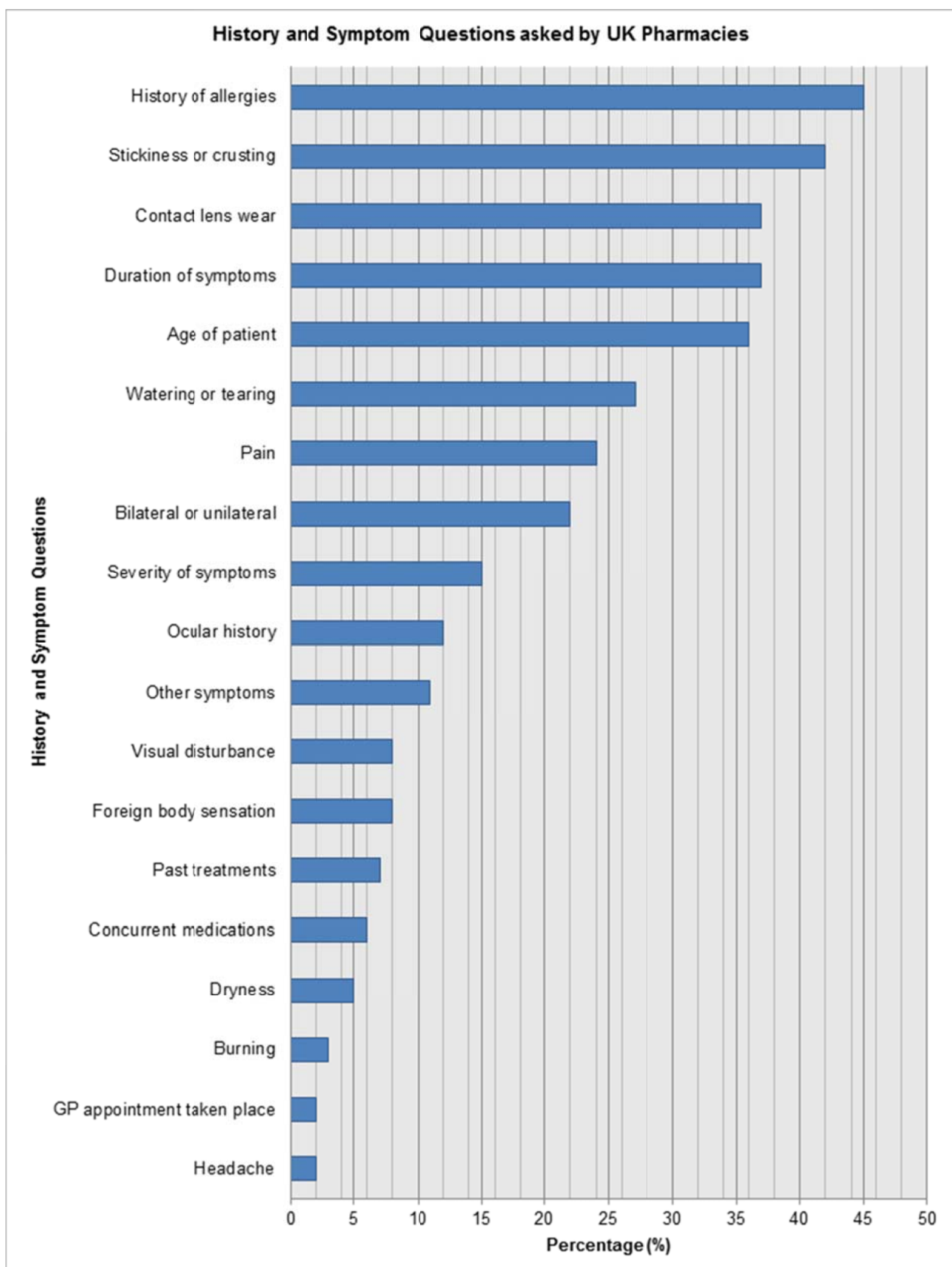

Figure 1: History and symptom questions asked by the pharmacy staff member $(n=100$ pharmacies). 
The majority of practices advised treatment $(91 \%, n=91)$, with most of these recommending pharmacological treatments $(96 \%, n=87)$ mainly in the form of topical eye drops $(97 \%$, $n=84)$. Four percent $(n=4)$ of the treatments were non-pharmacological, and included allergen avoidance and use of hypromellose. Those pharmacies that did not recommend treatment $(9.0 \%, n=9)$ referred the patient to their general practitioner $(n=4)$ or pharmacist $(n=4)$ but only two referred to an optometrist. The treatments advised by the pharmacies are shown in figure 2. 


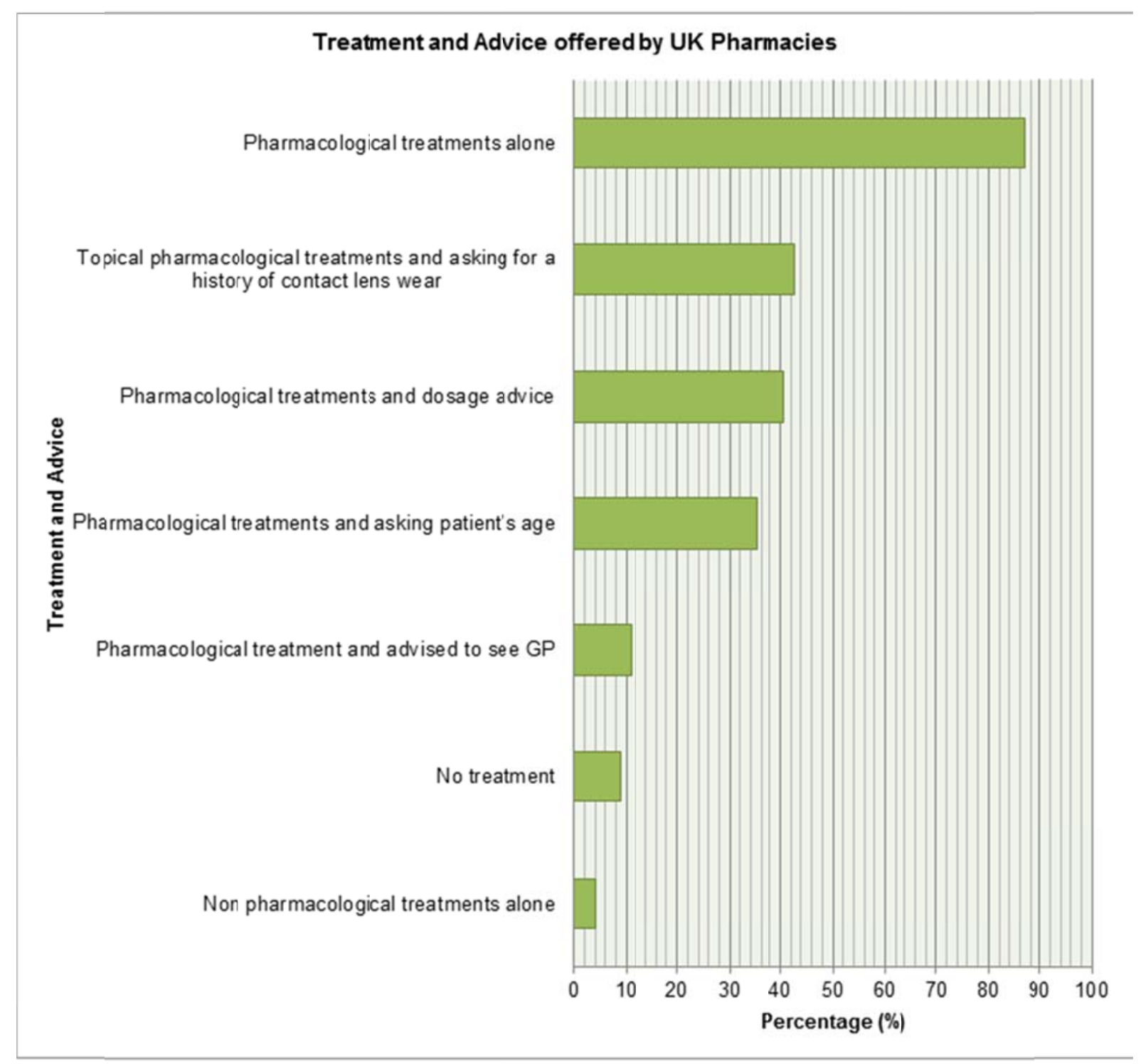

Figure 2: Treatment recommendations and management advice given by the pharmacy staff member ( $n=91$ pharmacies). 


\section{Discussion}

A mystery shopper technique was used to simulate a realistic consultation to determine the history and symptoms questioning of a patient with presumed allergic conjunctivitis and management recommended by community pharmacies across the UK. Pharmacies were not informed that the study was being conducted, thus eliminating the possibility of the Hawthorne effect influencing the outcome of the consultation. Although some authors have suggested mystery shopping techniques are unethical, such criticisms relate to competitive intelligence gathering between competing businesses ${ }^{5}$. Patient satisfaction is insufficient to fully evaluate healthcare service provision and safety alone, as patients often lack the expertise to formulate criteria to measure performance ${ }^{6}$. In addition, some authors argue that it is impossible to fully evaluate healthcare without using deceptive methods ${ }^{7}$. Analysis of patient records may be used to evaluate diagnostic and management performance, but pharmacies do not generally offer private consultations, or keep patient records. Mystery shopping techniques have previously been employed extensively in healthcare research to evaluate provision of specific services, but none have investigated the management of eye disease, such as ocular allergy, in community pharmacies.

The low number of questions asked on average in the present study are consistent with the low number of criteria covered on average by pharmacists when diagnosing ocular allergy in the study by Wolffsohn $(2009)^{3}$. The high proportion of pharmacies suggesting treatments compared to referral implies that pharmacy staff are confident in their abilities to diagnose and treat allergic conjunctivitis. This is supported by very few pharmacies advising referral to another healthcare professional to confirm diagnosis or for further investigation $(14 \%, n=13)$ and is consistent with a previous study ${ }^{3}$ where all pharmacists recommended treatment. Perhaps pharmacy staff perceive ocular symptoms as part of a systemic allergy rather than as a uniquely sensitised tissue. Despite their advanced instrumentation to examine the eyes to allow differential diagnosis when symptomology is not conclusive, only 2 pharmacies in this study recommended referral to an optometrist. This may be due to a lack of awareness 
by the public and other healthcare professionals of the abilities and role of optometrists in primary eye care. Conversely, optometrists may not be promoting or communicating their abilities effectively.

Preventing or minimising exposure to the causative allergen prevents the development of a hypersensitivity response ${ }^{1,8}$. However, allergen avoidance advice was given in less than $2 \%$ of pharmacies which concurs with a previous study with UK pharmacists ${ }^{3}$. Half the pharmacies advised the use of sodium cromoglycate $2 \%$, whose efficacy compared to placebo in the treatment of allergic conjunctivitis has been demonstrated in both environmental studies and conjunctival challenge models ${ }^{1,8}$. However, their mechanism of action result in a significant delay of up to several weeks in treating ocular allergy and an antihistamine would be more appropriate for initial symptomology management ${ }^{1}$. Interestingly, none of the pharmacies recommended lodoxamide trometamine $0.1 \%$, a mast cell stabiliser which produces a greater and faster clinical improvement in ocular allergy than sodium cromoglycate $2 \%{ }^{9}$. The second most common treatment was topical witch hazel (hamamelis virginiana), a plant extract with purported astringent properties. However, there is no evidence in the scientific literature regarding its pharmacological action or its efficacy in treating ocular allergies.

The vasoconstrictor naphazoline hydrochloride has been shown to be more effective in treating allergic conjunctivitis than a placebo ${ }^{10}$. However, there appears to be no scientific literature on xylometazoline-antazoline in treating allergic conjunctivitis, although one study found mild vasoconstriction of conjunctival blood vessels in the eyes of 16 healthy volunteers ${ }^{11}$. Of concern is that only one of the pharmacies recommending these products asked the patient's age or whether they had concurrent medical conditions or eye disease. Topical sympathomimetics are contraindicated in patients with glaucoma and should be used with caution in patients with heart disease, high blood pressure and diabetes ${ }^{8}$. 
Oral antihistamines are indicated where systemic allergies are present such as hay fever in addition to ocular signs and symptoms; although they are not as effective as topical antiallergic medications in treating allergic conjunctivitis, efficacy improves when they are combined $^{8}$. Hypromellose, an artificial tear supplement, may aid removal of allergens from the ocular surface and act as barrier to further allergen exposure ${ }^{8}$. Cooled artificial tears and cold compresses may also cause vasoconstriction of the conjunctival blood vessels and bring about symptomatic relief ${ }^{8}$. However, despite several authors recommending their use, scientific evidence is lacking. Incorrect treatment advice was low $(6 \%, n=6)$ where the antibacterial brolene (propamidine isetionate $0.1 \%$ ) was recommended. However, only a third of these pharmacies asked if the patient had stickiness or crusting of the eyelids/lashes and if they had any discharge from the eye, key questions in the differential diagnosis between bacterial and allergic conjunctivitis ${ }^{1,2,4}$.

Less than half of pharmacies recommending treatment asked about contact lens wear (41\%, $\mathrm{n}=37$ ). Topical administration of medications requires removal of contact lenses as the active ingredients and preservatives can bind to the contact lens and prolong exposure which may result in ocular toxicity or drug-induced allergic inflammation ${ }^{2}$

Hence there is a need for improved ophthalmology training and support for ocular allergy management in community pharmacy, as treatments can be purchased without prescription. If the diagnosis is in doubt, pharmacists should refer patients with suspected ocular allergy to healthcare professionals with equipment available to examine the eyes, such as optometrists. If pharmacological treatments are advised, only those which demonstrate efficacy should be recommended on an individual patient basis. 


\section{Conclusions.}

The differential diagnosis questioning and management of allergic conjunctivitis by community pharmacies in the UK is currently lacking and more training is warranted.

\section{Acknowledgements}

None

\section{Funding}

None

\section{Conflicts of Interest}

The authors have no financial or commercial conflicts of interest relating to this study. 


\section{References}

1. Abelson MB, Leonardi A, Smith L. The mechanisms, diagnosis and treatment of allergy. Rev Ophth. 2002; 9(4): 74-84.

2. Bielory L. Ocular allergy overview. Immunol Allergy Clin North Am. 2008 Feb; 28(1): 1-23.

3. Wolffsohn, J. S. The development of specialist ocular allergy services in UK optometric practice. MBA dissertation, Aston University. 2009.

4. Bilkhu PS, Wolffsohn JS, Naroo SA. A review of non-pharmacological and pharmacological management of seasonal and perennial allergic conjunctivitis. Contact Lens Ant Eye. 2012; 35: 9-16.

5. Shing MNK, Spence LJ. Investigating the limits of competitive intelligence gathering: is mystery shopping unethical? BE: ER. 2002; 11(4): 343-353.

6. Moriarty $\mathrm{H}$, McLeod D, Dowell A. Mystery shopping in health service evaluation. $\mathrm{Br} \mathrm{J}$ Gen Prac. 2003 Dec; 53(497): 942-946.

7. Walker T, George S. Mystery shopping in psychiatric service evaluation. The Psychiatrist. 2010; 34: 121-122.

8. Bielory L. Ocular allergy treatment. Immunol Allergy Clin North Am. 2008 Feb; 28(1): 189224.

9. Fahy GT, Easty DL, Collum LM, Benedict-Smith A, Hillery M, Parsons DG. Randomised double masked trial of lodoxamide and sodium cromoglycate in allergic eye disease. Eur $\mathrm{J}$ Ophthalmol. 1992 July-Sep; 2; 144.

10. Miller J, Wolf EH. Antazoline phosphate and naphazoline hydrochloride, singly and in combination for the treatment of allergic conjunctivitis - a controlled, double blind clinical trial. Ann Allergy. 1975 Aug; 35(2); 81-86. 
11. Trew DR, Wright LA, Smith SE. Otrivine-antistin - pupil, corneal and conjunctival responses to topical administration. Eye. 1989; 3: 294-297. 